\title{
Scoliosis may increase the risk of recurrence of lumbar disc herniation after microdiscectomy
}

\author{
Hsuan-Kan Chang, MD, ${ }^{1,2}$ Huang-Chou Chang, MD, ${ }^{5}$ Jau-Ching Wu, MD, PhD, 1,2 \\ Tsung-Hsi Tu, MD, ${ }^{1,2}$ Li-Yu Fay, MD, 1,2 Peng-Yuan Chang, MD, ${ }^{1,2}$ Ching-Lan Wu, MD, ${ }^{2,4}$ \\ Wen-Cheng Huang, MD, PhD, ${ }^{1,2}$ and Henrich Cheng, MD, PhD ${ }^{1-3}$

\begin{abstract}
${ }^{1}$ Department of Neurosurgery, Neurological Institute, and ${ }^{4}$ Department of Radiology, Taipei Veterans General Hospital; ${ }^{2}$ School of Medicine and ${ }^{3}$ Institute of Pharmacology, National Yang-Ming University, Taipei; and ${ }^{5}$ Department of Surgery, Kaohsiung Veterans General Hospital, Kaohsiung, Taiwan
\end{abstract}

OBJECT The aim of this paper was to investigate the risk of recurrence of lumbar disc herniation (LDH) in patients with scoliosis who underwent microdiscectomy.

METHODS A series of consecutive patients who underwent microdiscectomy for LDH was retrospectively reviewed. The inclusion criteria were young adults younger than 40 years who received microdiscectomy for symptomatic 1-level $\mathrm{LDH}$. An exclusion criterion was any previous spinal surgery, including fusion or correction of scoliosis. The patients were divided into 2 groups: those with scoliosis and those without scoliosis. The demographic data in the 2 groups were similar. All medical records and clinical and radiological evaluations were reviewed.

RESULTS A total of 58 patients who underwent 1-level microdiscectomy for LDH were analyzed. During the mean follow-up of 24.6 months, 6 patients (10.3\%) experienced a recurrence of LDH with variable symptoms. The recurrence rate was significantly higher among the scoliosis group than the nonscoliosis group ( $33.3 \%$ vs $2.3 \%, p=0.001)$. Furthermore, the recurrence-free interval in the scoliosis group was short.

CONCLUSIONS Young adults (< 40 years) with uncorrected scoliosis are at higher risk of recurrent LDH after microdiscectomy.

http://thejns.org/doi/abs/10.3171/2015.7.SPINE15133

KEY WORDS lumbar disc herniation; recurrence; scoliosis; microdiscectomy

$\mathrm{L}$ UMBAR disc herniation (LDH) causing sciatica is one of the most common spinal conditions requiring surgery in neurosurgical practice. Although LDH can often be managed conservatively, resection of the sequestrated disc is usually reserved for symptomatic patients whose condition is refractory to medical treatment for a prolonged time. Also, there are many surgical approaches noted in the literature that have demonstrated effectiveness for the treatment of LDH. In past decades, microdiscectomy through a tubular or expandable retractor has been reported to be efficacious and has become a popular surgical technique. ${ }^{22}$ This minimally invasive approach is not only clinically effective but also economically cost-effective. ${ }^{16}$
Most of the LDH patients who received microdiscectomy had satisfactory results. ${ }^{14}$ However, a portion $(5 \%-15 \%)$ of patients will develop LDH recurrence, even after a very successful discectomy. ${ }^{4,5,20}$ In the literature, it has been reported that the risk factors of recurrent LDH included male sex, old age, cigarette smoking, trauma, diabetes, high body mass index (BMI), occupational heavy weight lifting, and degenerative disc disease..$^{2,3,14,15,20}$ Furthermore, the degree of annular competence, type of herniation, and protrusion type of LDH have been demonstrated to increase the risk of the recurrence of $\mathrm{LDH} .{ }^{10}$

Patients with adolescent idiopathic scoliosis have altered biomechanics in the lower lumbar spine.,13 It is not clear whether this change in weight bearing causes a high- 
er incidence of LDH. ${ }^{1}$ The effect of scoliosis in terms of risk of LDH recurrence is unknown. Therefore, the optimal management of LDH in scoliosis patients remains uncertain. ${ }^{8}$

The current study aimed to investigate the differences in recurrence rates of LDH between patients who had scoliosis and those who did not. To date, there has been a paucity of data on the effect of microdiscectomy in patients with adolescent idiopathic scoliosis, and, to our knowledge, no reports have addressed this issue.

\section{Methods}

\section{Inclusion Criteria of Patients}

This retrospective study included consecutive patients who had single-level LDH proven by advanced imaging studies (MRI or CT) with corresponding symptoms and signs such as low-back pain, neurogenic claudication, radiculopathy, or a positive straight leg-raising test. All patients received medical treatment for at least 6 weeks before undergoing surgery and had no other comorbidities contraindicating surgery. Exclusion criteria were prior lumbar surgery, thoracolumbar fractures, spondylolisthesis, spinal infection, tumor, inflammatory spondyloarthropathy, and pregnancy. Patients older than 40 years were also excluded to mostly eliminate degenerative scoliosis and spondylosis-related kyphotic deformity.

The patients were divided into 2 groups: those with scoliosis and those without scoliosis. The identification of scoliosis was made prior to surgery, but was not considered a preclusion for surgery, described in the following paragraph. All demographic data and perioperative records were reviewed and compared between the 2 groups. Every patient underwent follow-up for more than 2 years with both clinical and radiographic evaluations.

\section{Surgical Techniques}

In the current series, every operation was performed using the same surgical technique, standard microdiscectomy, which was similar to that first described by Williams in $1978,{ }^{22}$ with various modifications over time. ${ }^{6,9,12,18}$ In this series, the operative level was identified using intraoperative fluoroscopy. Subsequently, a skin incision of about $3 \mathrm{~cm}$ was made close to the midline, and the fascia and muscles were split until the hemilamina was exposed. Through a tubular or expandable retractor, under the microscope, laminotomy was carried out using high-speed drills and Kerrison rongeurs. Decompression of the neuroforamen was achieved after excision of the ligamentum flavum and surrounding osteophytes. Discectomy was then performed through this window, with the nerve root retracted medially when needed. Unless an obviously ruptured disc or a sequestrated disc fragment was met, a small incision was made with a sharp knife over the annulus fibrosus of the intervertebral disc.

Loose disc fragments were removed using a curette and disc rongeurs. In the few instances in which a dura tear and CSF leakage were encountered, primary repair with suture and tissue glue sealants was performed. The muscles and fascia were then closed tightly before skin closure. For an initial postoperative period of time, we encouraged early ambulation but with the avoidance of bending, heavy weight lifting, and excessive exercising.

\section{Clinical and Radiological Evaluations}

All patients' data, including charts, operative notes, clinical follow-up reports, subsequent telephone interviews, and radiographic images were reviewed. Standardized clinical outcome measurements, such as the visual analog scale (VAS) score, were collected during clinic visits at each time point of follow-up, at approximately 6 weeks and 3,6, 12, and 24 months, by 2 special nurse assistants under the physicians' supervision.

All patients underwent screening radiographs of the entire spine prior to the operation. Identification of scoliosis was confirmed by radiologists. Whether the patient had thoracolumbar scoliosis did not affect the decision for microdiscectomy. Recurrence of LDH was assessed by clinical evaluation or radiological studies (i.e., MRI) during the follow-up. Confirmation of recurrence had to meet both of the following criteria: 1) recurrent symptoms or signs, such as neurogenic claudication and/or radiculopathy, that could be correlated to the LDH after a symptomfree postoperation interval; and 2) radiographic evidence of LDH on CT scan or MRI at the same level to previous surgery but morphologically different (Fig. 1).

All related risk factors reported in the literature, including smoking, diabetes, sex, trauma, occupational heavy weight lifting, and BMI were compared between the scoliosis and nonscoliosis groups.

\section{Statistical Analysis}

Using SPSS software (SPSS Inc.), independent t-tests and paired t-tests were applied for analysis of continuous variables, and the Pearson chi-square test was used for categorical variables. Statistical significance was set at $p$ $<0.05$.

\section{Results}

A total of 58 consecutive patients were analyzed in the current series with a mean follow-up period of 24.6 months. Fifteen patients (25.8\%) comprised the scoliosis group, and 43 (74.2\%) comprised the nonscoliosis group. Most instances of LDH occurred at the L5-S1 $(55.1 \%, \mathrm{n}=$ $32)$ and $\mathrm{L} 4-5(43.1 \%, \mathrm{n}=25)$ levels; only 1 instance $(1.7 \%)$ occurred at the L3-4 level.

All patients had symptom relief after the operation, and significantly decreased VAS scores were demonstrated overall (Fig. 2). When comparing the scoliosis and nonscoliosis groups, there were no differences in demographic data or the risk factors of recurrent LDH, including sex, age, BMI, cigarette smoking, diabetes, traumatic events, and occupational heavy weight lifting (Table 1). There was 1 intraoperative dural tear in the scoliosis group; there were no other surgical complications in the other patients (Table 2).

Among the 15 patients in the scoliosis group, 5 patients (33.3\%) experienced a recurrence of $\mathrm{LDH}$ at an average of 5.5 months postoperatively, at the time of diagnosis. On the other hand, only 1 of 43 patients $(2.3 \%)$ in the nonscoliosis group had a recurrence of LDH. A significantly 


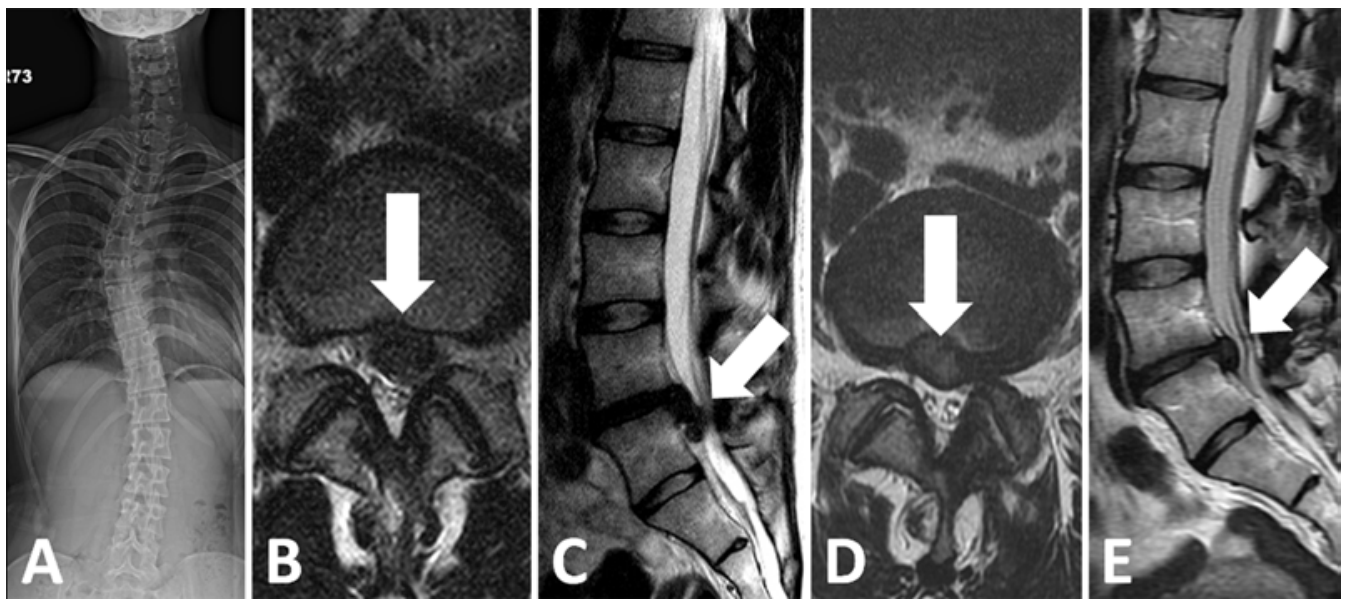

FIG. 1. Images obtained in a 29-year-old woman with L5-S1 LDH and scoliosis who experienced a relapse of symptoms 4 months after microdiscectomy. The different contours of bulging masses on the preoperative and follow-up MR images indicate a recurrence of LDH. A: Anteroposterior radiograph of the thoracolumbar spine. B and C: Preoperative axial and sagittal T2-weighted images at L5-S1. The arrows indicate the LDH. D and E: Follow-up axial and sagittal T2-weighted images at L5-S1. The arrows indicate the recurrent LDH.

higher rate of recurrence was demonstrated in the scoliosis group $(33.3 \%$ vs $2.3 \%, \mathrm{p}=0.001)$ (Table 3$)$. All patients identified for recurrent LDH were symptomatic and were initially managed with medication and rehabilitation. In the scoliosis group, 2 patients $(40 \%)$ did not improve with conservative treatment and subsequently received secondary surgery. On the other hand, in the nonscoliosis group, the only patient with recurrent LDH eventually received secondary surgery (Fig. 3). The only patient who had a recurrence of $\mathrm{LDH}$ in the nonscoliosis group had a recurrence-free interval of 39 months, whereas the recurrencefree interval for the scoliosis group was 5.5 months (range 2-12 months; Fig. 4).

\section{Discussion}

Lumbar disc surgery is one of the most commonly performed surgical procedures in the United States, with more than 200,000 cases performed per year. ${ }^{21}$ Recurrence of LDH after the first surgery is not uncommon and can adversely affect the patient's quality of life. ${ }^{16,21}$ In the current series of 58 consecutive patients with $\mathrm{LDH}$, initially

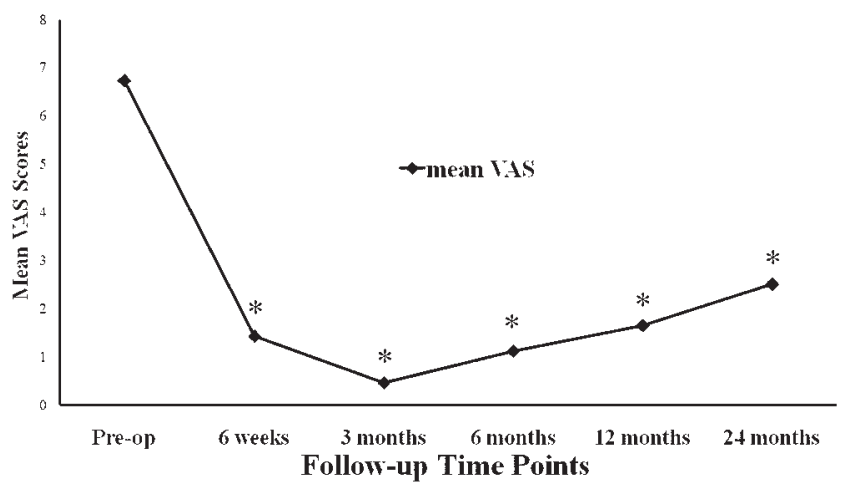

FIG. 2. Mean VAS scores of patients in the series at preoperation and at each follow-up time point. There was significant improvement at each of the follow-up time points after surgery when compared with preoperative scores. ${ }^{*} p<0.05$ compared with preoperative scores.
$100 \%$ experienced symptom relief after microdiscectomy. Their preoperative VAS score improved significantly after the operation. However, 6 patients $(10.3 \%)$ who were free of symptoms after microdiscectomy later had a relapse of symptoms caused by recurrent LDH at the operated level. Interestingly, most of these patients $(83.3 \%, 5$ of 6$)$ who had recurrent $\mathrm{LDH}$ also had thoracolumbar scoliosis. Patients in the scoliosis group had a significantly higher rate of recurrent $\mathrm{LDH}$ than those in the nonscoliosis group $(33.3 \%$ vs $2.3 \%, p=0.001)$. Overall, patients with scoliosis had a greater propensity for a secondary discectomy, although statistical significance was not reached $(13.3 \%$ vs $2.3 \%, \mathrm{p}=0.097)$.

In summary, although microdiscectomy for patients with thoracolumbar scoliosis yields comparably good effects for symptom relief for $\mathrm{LDH}$, there is a significantly higher rate of recurrence within the 1st year after surgery.

In the current study, most $(89.7 \%)$ of the patients remained symptom free after microdiscectomy for LDH throughout the follow-up. The recurrence rate of $10.3 \%$ $(6 / 58)$ was compatible with that in most of the published series of surgery for LDH, despite various surgical techniques. ${ }^{20,21}$ The true etiology of recurrence after successful discectomy remains unclear. There were several risk factors reportedly associated with recurrence of $\mathrm{LDH}$, in-

TABLE 1. Demographic data in all patients

\begin{tabular}{lccc}
\hline \multicolumn{1}{c}{ Variable } & $\begin{array}{c}\text { Scoliosis Group } \\
(\mathrm{n}=15)\end{array}$ & $\begin{array}{c}\text { Nonscoliosis } \\
\text { Group }(\mathrm{n}=43)\end{array}$ & $\begin{array}{c}\mathrm{p} \\
\text { Value }\end{array}$ \\
\hline No. of females & $7(46.6 \%)$ & $11(25.5 \%)$ & 0.116 \\
\hline Median age at op in yrs & 28.1 & 30.9 & 0.143 \\
\hline Mean BMI \pm SD & $28.13 \pm 6.66$ & $25.08 \pm 3.59$ & 0.902 \\
\hline Diabetes & $0(0 \%)$ & $1(2.3 \%)$ & 0.556 \\
\hline Smoking & $5(33 \%)$ & $8(18.6 \%)$ & 0.171 \\
\hline Traumatic event & $2(13.3 \%)$ & $9(20.9 \%)$ & 0.541 \\
\hline Heavy weight bearing & $2(13.3 \%)$ & $9(20.9 \%)$ & 0.541 \\
\hline Mean follow-up in mos & 23.1 & 25.1 & \\
\hline
\end{tabular}


TABLE 2. Surgery-related complications

\begin{tabular}{lcc}
\hline \multicolumn{1}{c}{ Complication } & Scoliosis Group & Nonscoliosis Group \\
\hline Dural tear & 1 & 0 \\
\hline Infection & 0 & 0 \\
\hline Cauda equina syndrome & 0 & 0 \\
\hline Drop foot & 0 & 0 \\
\hline
\end{tabular}

cluding male sex, cigarette smoking, traumatic events, "incompetent" annulus fibrosus, high BMI, diabetes, and occupational heavy weight lifting. ${ }^{2,3,10,14,15,20}$ However, there are controversies among these series. For example, Suk et al. ${ }^{20}$ and Miwa et al..$^{14}$ demonstrated that cigarette smoking increased risks of recurrent LDH, but Swartz and Trost ${ }^{21}$ did not concur. Likewise, Kim et al. ${ }^{10}$ reported that high BMI could cause recurrence of $\mathrm{LDH}$, whereas Moliterno et al. ${ }^{16}$ demonstrated that lower BMI was associated with a greater risk of recurrence. In Moliterno's series, there was a lower mean BMI in the recurrence group than the nonrecurrence group (24.9 vs 28.3$).^{16}$

It is reasonable to infer that patients with thoracolumbar scoliosis would have altered biomechanical wearing in their lumbar intervertebral discs. Ozgen et al. ${ }^{17}$ reported that $82 \%$ of adolescent patients with $\mathrm{LDH}$ had scoliosis. However, the actual causal effect of scoliosis in LDH has not been established. Only case reports exist in the literature to associate scoliosis with LDH. ${ }^{11,19}$ It is still uncertain about the mechanism and frequency of LDH in patients with scoliosis. This study therefore attempted to demonstrate for the first time the association between scoliosis and recurrent LDH in young adults. There were 15 patients in the current study who had scoliosis and underwent microdiscectomy without previous or later corrective surgery for scoliosis. This substantial portion (25.8\%) of young adult patients with scoliosis implies that scoliosis could be a risk factor of LDH. The rates of recurrence of LDH were even more discrepant (33.3\% vs $2.3 \%$ ) between the nonscoliosis and scoliosis patients, respectively. Nevertheless, the association between LDH and scoliosis requires a larger number of patients to corroborate.

Management of recurrent LDH after surgical discectomy varies tremendously. There is a wide range of treatment options, including observation, pharmacological therapy, physical rehabilitation, and reoperation..$^{21}$ The surgical options also vary among experts, such as endoscopic/open discectomy, redoing microdiscectomy, or spinal instruments. The optimal management for recurrent LDH in patients with scoliosis has been infrequently

TABLE 3. Recurrence rate and recurrence with second operation between the scoliosis and nonscoliosis groups

\begin{tabular}{lccc}
\hline \multicolumn{1}{c}{ Variable } & $\begin{array}{c}\text { Scoliosis Group } \\
(\mathrm{n}=15)\end{array}$ & $\begin{array}{c}\text { Nonscoliosis } \\
\text { Group }(\mathrm{n}=43)\end{array}$ & $\mathrm{p}$ Value \\
\hline Recurrence & $5(33.33 \%)$ & $1(2.32 \%)$ & $0.001^{*}$ \\
\hline Recurrence w/ 2nd op & $2(13.33 \%)$ & $1(2.32 \%)$ & 0.097 \\
\hline
\end{tabular}

\footnotetext{
* Statistically significant
}

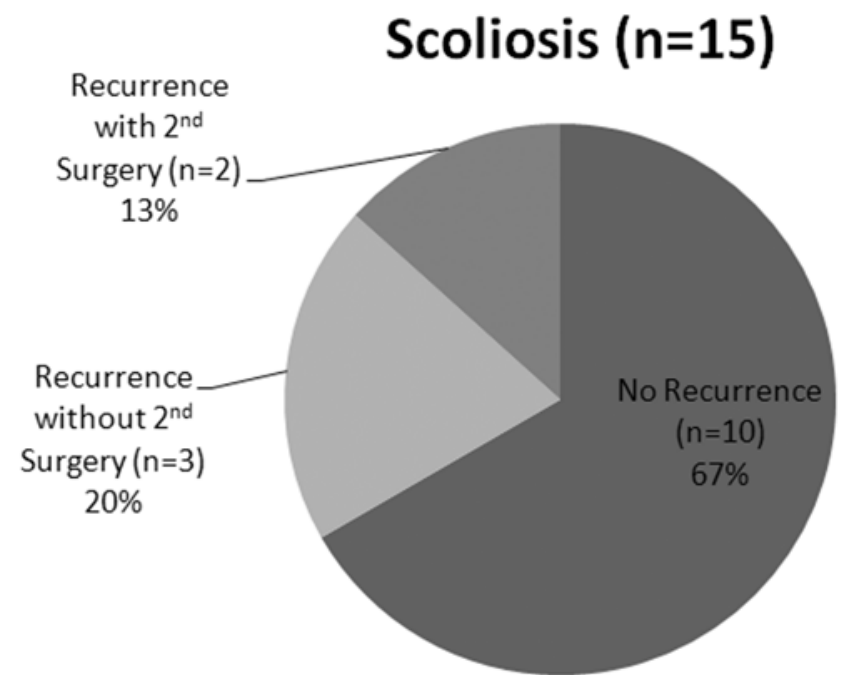

\section{Non-Scoliosis $(n=43)$}

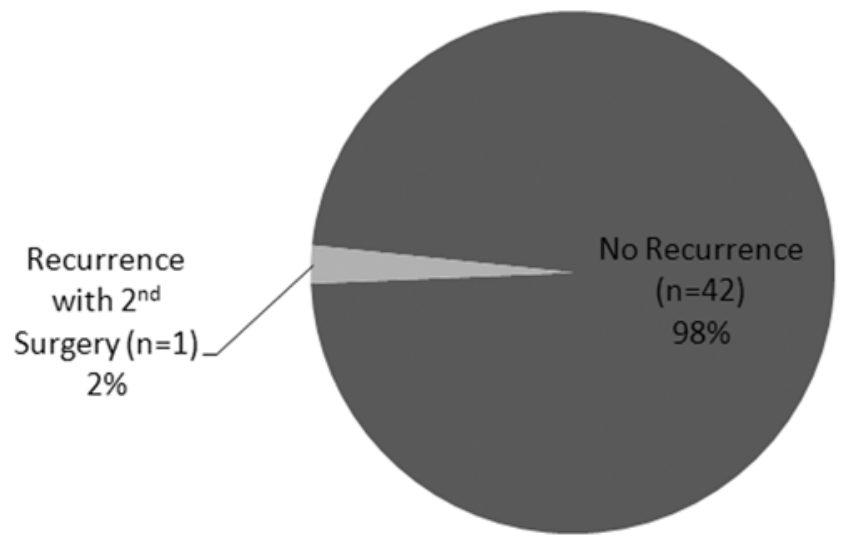

FIG. 3. Pie chart of percentages of nonrecurrence, recurrence without reoperation, and recurrence with reoperation, within both groups (the scoliosis and nonscoliosis groups, respectively).

reported. In the current study, all 5 patients with recurrent LDH were treated initially with medication and physical rehabilitation. Subsequently, 2 underwent reoperation because of refractory symptoms. However, the optimal strategy and timing of intervention for these cases of recurrent LDH remain elusive.

There are limitations to the current study. This was a relatively small cohort of patients with LDH. This series retrospectively analyzed 58 consecutive young adults $(<$ 40 years) with LDH who underwent microdiscectomy. Thus, the strict inclusion/exclusion criteria, which tended to eliminate other confounding factors (e.g., degenerative scoliosis, spondylosis, and spondylolisthesis), unfortunately also reduced the number of patients, which could have been substantially increased if the study had involved older patients. However, this design of the study reduced the effect of degeneration in these degenerative disc diseases, and yielded a more homogeneous cohort for evaluation (Table 1). Furthermore, this study had an average follow-up of slightly longer than 2 years, which might not be adequate for those late recurrences of LDH. However, 

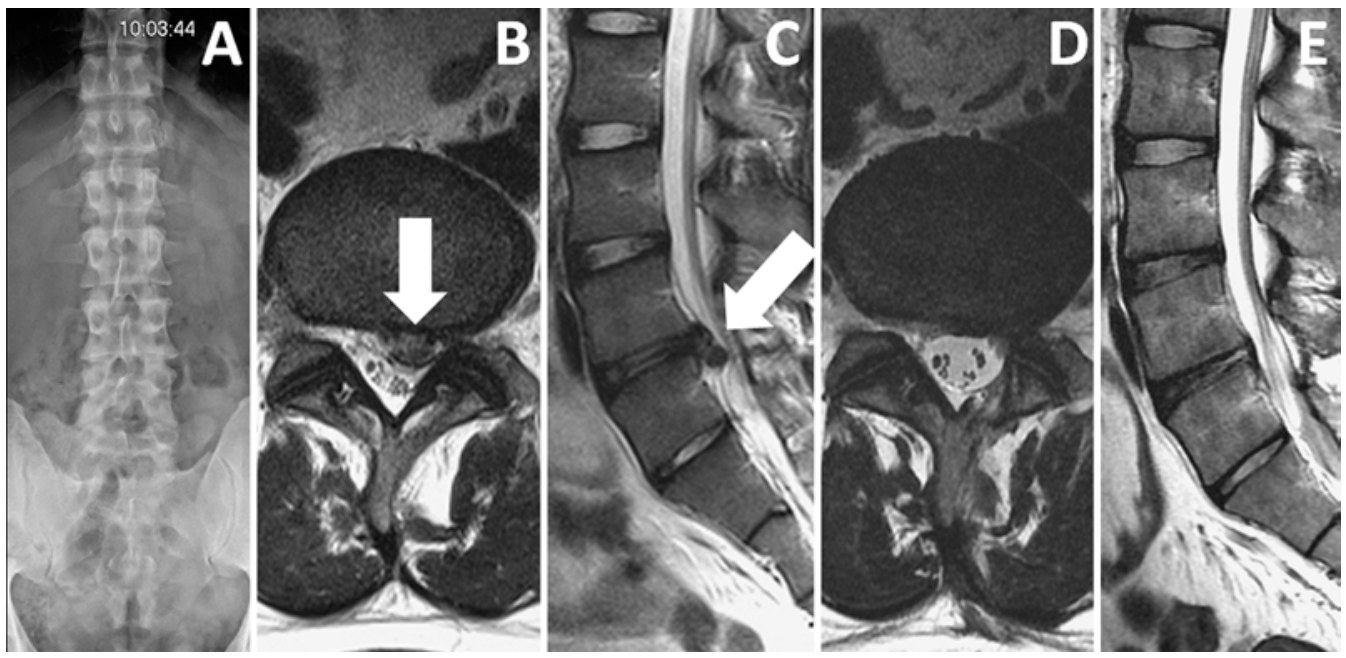

FIG. 4. Images obtained in a 26-year-old man with L5-S1 LDH and no scoliosis. This patient remained asymptomatic to date and the follow-up MR images were obtained 1 year after the microdiscectomy. A: Anteroposterior radiograph of the thoracolumbar spine. B and C: Preoperative axial and sagittal T2 weighted-images at L5-S1. The arrows indicate the LDH. D and E: Follow-up axial and sagittal T2-weighted images at L5-S1.

the follow-up rate was extremely high in the current study and the duration was already adequate for the detection of significance. Nevertheless, the relatively early recurrence of LDH in patients with scoliosis warrants future studies with longer follow-up in a larger cohort.

\section{Conclusions}

Young adults $(<40$ years) with scoliosis are at higher risk of recurrent $\mathrm{LDH}$ after microdiscectomy than patients without scoliosis. Although some of the recurrences may be asymptomatic, patients with scoliosis should be aware of this risk before they undergo microdiscectomy for $\mathrm{LDH}$.

\section{References}

1. Brox JI, Lange JE, Steen H: Comorbidity influenced healthrelated quality of life of 390 patients with idiopathic scoliosis at long-term follow-up. Eur J Phys Rehabil Med 50:73-81, 2014

2. Carragee EJ, Han MY, Suen PW, Kim D: Clinical outcomes after lumbar discectomy for sciatica: the effects of fragment type and anular competence. J Bone Joint Surg Am 85A:102-108, 2003

3. Cinotti G, Roysam GS, Eisenstein SM, Postacchini F: Ipsilateral recurrent lumbar disc herniation. A prospective, controlled study. J Bone Joint Surg Br 80:825-832, 1998

4. Connolly ES: Surgery for recurrent lumbar disc herniation. Clin Neurosurg 39:211-216, 1992

5. Fandiño J, Botana C, Viladrich A, Gomez-Bueno J: Reoperation after lumbar disc surgery: results in 130 cases. Acta Neurochir (Wien) 122:102-104, 1993

6. Foley KT, Smith MM, Rampersaud YR: Microendoscopic approach to far-lateral lumbar disc herniation. Neurosurg Focus 7(5): e5, 1999

7. Hristova GI, Jarzem P, Ouellet JA, Roughley PJ, Epure LM, Antoniou J, et al: Calcification in human intervertebral disc degeneration and scoliosis. J Orthop Res 29:1888-1895, 2011
8. Jehan S, Elsayed S, Webb J, Boszczyk B: L5-S1 disc replacement after two previous fusion surgeries for scoliosis. Eur Spine J 20:669-673, 2011

9. Khoo LT, Fessler RG: Microendoscopic decompressive laminotomy for the treatment of lumbar stenosis. Neurosurgery 51 (5 Suppl):S146-S154, 2002

10. Kim JM, Lee SH, Ahn Y, Yoon DH, Lee CD, Lim ST: Recurrence after successful percutaneous endoscopic lumbar discectomy. Minim Invasive Neurosurg 50:82-85, 2007

11. Krishnan KM, Newey ML: Lumbar scoliosis associated with a disc herniation in an adult. Rheumatology (Oxford) 40:1427-1428, 2001

12. Maroon JC: Current concepts in minimally invasive discectomy. Neurosurgery 51 (5 Suppl):S137-S145, 2002

13. Meir A, McNally DS, Fairbank JC, Jones D, Urban JP: The internal pressure and stress environment of the scoliotic intervertebral disc - a review. Proc Inst Mech Eng H 222:209-219, 2008

14. Miwa S, Yokogawa A, Kobayashi T, Nishimura T, Igarashi $\mathrm{K}$, Inatani $\mathrm{H}$, et al: Risk factors of recurrent lumbar disc herniation: a single center study and review of the literature. J Spinal Disord Tech 28:E265-E269, 2015

15. Mobbs RJ, Newcombe RL, Chandran KN: Lumbar discectomy and the diabetic patient: incidence and outcome. J Clin Neurosci 8:10-13, 2001

16. Moliterno JA, Knopman J, Parikh K, Cohan JN, Huang QD, Aaker GD, et al: Results and risk factors for recurrence following single-level tubular lumbar microdiscectomy. J Neurosurg Spine 12:680-686, 2010

17. Ozgen S, Konya D, Toktas OZ, Dagcinar A, Ozek MM: Lumbar disc herniation in adolescence. Pediatr Neurosurg 43:77-81, 2007

18. Palmer S: Use of a tubular retractor system in microscopic lumbar discectomy: 1 year prospective results in 135 patients. Neurosurg Focus 13(2):E5, 2002

19. Pinto FC, Poetscher AW, Quinhones FR, Pena M, Taricco MA: Lumbar disc herniation associated with scoliosis in a 15-year-old girl: case report. Arq Neuropsiquiatr 60 (2A):295-298, 2002

20. Suk KS, Lee HM, Moon SH, Kim NH: Recurrent lumbar disc herniation: results of operative management. Spine (Phila Pa 1976) 26:672-676, 2001 
21. Swartz KR, Trost GR: Recurrent lumbar disc herniation. Neurosurg Focus 15(3):E10, 2003

22. Williams RW: Microlumbar discectomy: a conservative surgical approach to the virgin herniated lumbar disc. Spine (Phila Pa 1976) 3:175-182, 1978

\section{Disclosures}

The authors report no conflict of interest concerning the materials or methods used in this study or the findings specified in this paper.

\section{Author Contributions}

Conception and design: all authors. Acquisition of data: all authors. Analysis and interpretation of data: all authors. Draft- ing the article: JC Wu, HK Chang, HC Chang, Tu, PY Chang, CL Wu. Critically revising the article: JC Wu, HK Chang, HC Chang, Tu, PY Chang, Huang, Cheng. Reviewed submitted version of manuscript: JC Wu, HK Chang, HC Chang, Tu, PY Chang, Huang, Cheng. Approved the final version of the manuscript on behalf of all authors: JC Wu. Statistical analysis: JC Wu, HK Chang, HC Chang, Tu. Administrative/technical/ material support: JC Wu, HK Chang, HC Chang, CL Wu. Study supervision: JC Wu, HK Chang, HC Chang, Huang, Cheng.

\section{Correspondence}

Jau-Ching Wu, Department of Neurosurgery, Neurological Institute, Taipei Veterans General Hospital, Rm. 508, 17F, No. 201, Shih-Pai Rd., Sec. 2, Beitou, Taipei 11217, Taiwan. email: jauching@gmail.com. 